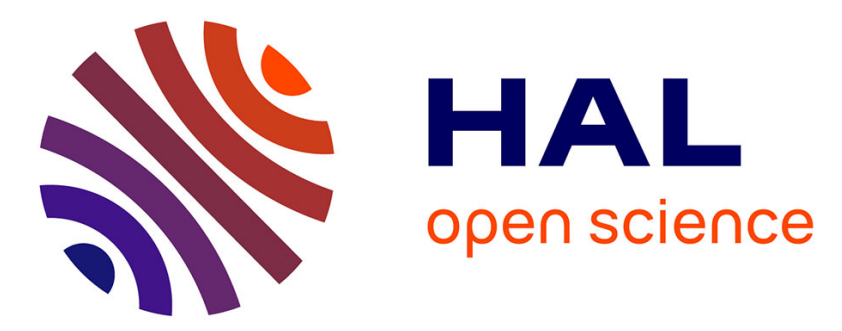

\title{
Blind Separation of Surface Electromyographic Mixtures from Two Finger Extensor Muscles
}

\author{
Anton Dogadov, Christine Serviere, Franck Quaine
}

\section{To cite this version:}

Anton Dogadov, Christine Serviere, Franck Quaine. Blind Separation of Surface Electromyographic Mixtures from Two Finger Extensor Muscles. LVA/ICA 2015 - 12th International Conference on Latent Variable Analysis and Signal Separation, Aug 2015, Liberec, Czech Republic. pp.481-488, 10.1007/978-3-319-22482-4_56. hal-01191243

\section{HAL Id: hal-01191243 \\ https://hal.science/hal-01191243}

Submitted on 1 Sep 2015

HAL is a multi-disciplinary open access archive for the deposit and dissemination of scientific research documents, whether they are published or not. The documents may come from teaching and research institutions in France or abroad, or from public or private research centers.
L'archive ouverte pluridisciplinaire HAL, est destinée au dépôt et à la diffusion de documents scientifiques de niveau recherche, publiés ou non, émanant des établissements d'enseignement et de recherche français ou étrangers, des laboratoires publics ou privés. 


\title{
Blind separation of surface electromyographic mixtures from two finger extensor muscles
}

\author{
Anton Dogadov, Christine Serviere, Franck Quaine \\ Univ. Grenoble Alpes, GIPSA-Lab, F-38000 Grenoble, France \\ CNRS, GIPSA-Lab, F-38000 Grenoble, France \\ \{anton. dogadov, christine. serviere, franck. quaine\} @gipsa- \\ lab.grenoble-inp.fr
}

\begin{abstract}
Blind source separation (BSS) was performed to reduce the crosstalk in the surface electromyografic signals (SEMG) for the muscle force estimation applications. A convolutive mixture model was employed to separate the SEMG signals from two finger extensor muscles using a frequency-domain approach. It was assumed that the tension of each muscle varies independently and the independence of the SEMG was replaced by minimization of the covariance of muscle forces represented by integrated SEMG. This covariance was also used to resolve the permutation ambiguity inherent to the frequencydomain BSS. The forces estimated by the reconstructed sources were compared with the measured forces to calculate the crosstalk reduction efficiency. The proposed algorithm was shown to be more effective in frequency domain than an ICA algorithm for extensor muscles crosstalk reduction.
\end{abstract}

Keywords. Blind source separation, convolutive mixture, surface electromyography, muscle crosstalk reducing.

\section{Introduction}

Surface electromyografic signals (SEMG) are widely used in medicine, prosthesis control and biomechanical studies [1]. Integrated SEMG (IEMG) is commonly used in biomechanics as an estimator of muscle force [2]. However, crosstalk or interference from neighbor muscles is a widespread problem in SEMG measurements [3]. It appears when two or more muscles situated close to each other are active during a SEMG recording. This effect may cause precision decrease of IEMG-based force estimations. Blind source separation (BSS) methods may be performed to reduce this crosstalk [2], [4] and thus improve the performance of muscle force estimation.

BSS is a method of source signal recovering from several mixtures when no a priori information is available on source properties (source spatial position etc.). To reduce the crosstalk from neighbor muscles BSS is applied to mixtures, in which each muscle is thought to be a source, and mixture signals are thought to be transformed vectors of source signals. Most of mixing transformations are supposed to be linear, instantaneous or convolutive. A linear instantaneous model can be used in the case of small muscles located close to each other [4]. However, validity of the instantaneity 
hypothesis is very sensitive to electrode location [2]. Merletti [5] explained the limitations of the instantaneous model by a convolutive effect of a volume conductor and by action potential propagation. Jiang and Farina [6] proposed an extension of a BSS technique based on second-order moments (SOBI) for the case of sources being delayed in the mixtures. However, little information is available on convolutive mixture separation of SEMG signals.

The purpose of this study was to perform convolutive BSS of SEMG mixtures to reduce the crosstalk in the SEMG signals and to improve the precision of the muscle force estimation. We first discus the hypothesis of independent sources for SEMG signals. As independence of the SEMG sources is not always verified, we propose to replace it by a criterion calculated on the integrated SEMG. The criterion characterizes the fact that the variations of the forces produced by the two muscles are biomechanically independent. This criterion was also used to resolve the permutation ambiguity inherent to the frequency-domain BSS.

\section{Blind source separation of SEMG signals}

\subsection{Mixture Model}

Let us consider the standard convolutive mixing model of two EMG signals:

$$
x_{k}(t)=\sum_{j=1}^{2} \sum_{l=0}^{L-1} h_{k j}(l) s_{j}(t-l),
$$

where $x_{1}(t), x_{2}(t)$ denote the observation mixtures, $s_{1}(t), s_{2}(t)$ the source signals. $h_{k j}(l)$ are the elements of the impulse response matrix from source $j$ to sensor $k, L$ is the length of the impulse response. A noise-free model was considered as the noise can be previously filtered before SEMG separation. The FIR convolutive mixing model can be reformulated into an instantaneous one in the frequency domain. N-point shorttime Fourier transform was performed (STFT), which transformed the observation sequences $\left\{x_{k}(t)\right\}$ to the time-frequency domain $\left\{X_{k}(t, f)\right\}$. For each frequency bin $f$ equation (1) becomes:

$$
X_{k}(t, f)=\sum_{j=1}^{2} H_{k j}(f) S_{j}(t, f),
$$

or in the matrix form:

$$
\mathbf{X}(t, f)=\mathbf{H}(f) \mathbf{S}(t, f)
$$




\subsection{Blind Source Separation of SEMG}

BSS is generally based on the assumption of independent sources and the idea is to adjust the separating filters $\mathbf{G}(f)$ such that the outputs $\mathbf{y}$ are as mutually independent as possible in each frequency $\operatorname{bin} f$ :

$$
\mathbf{Y}(t, f)=\mathbf{G}(f) \mathbf{X}(t, f),
$$

Usually in ICA algorithms, $\mathbf{G}(f)$ is decomposed as:

$$
\mathbf{Y}(t, f)=\mathbf{R}(f) \mathbf{V}(f) \mathbf{X}(t, f),
$$

where $\mathbf{R}$ is a rotation matrix, $\mathbf{V}$ is a whitening matrix. As sources are supposed to be uncorrelated, the whitening matrix is calculated by eigenvalue decomposition of the covariance matrix in each frequency. To calculate the rotation matrix $\mathbf{R}$ an independence criterion must be chosen, for example, with the help of fourth-order cumulants [7].

For SEMG signals, the hypothesis of mutually independent sources has to be discussed. A muscle is composed of Muscle Fibres (MFs) organized into Motor Units (MUs). This functional unit is composed of an alpha-motoneuron, innervating several MFs (from tens to hundreds MFs depending on the MU and on the muscle characteristics). Activation of the MU produces an electric field generated by each MF into the MU. The summation of these electric fields provides a specific waveform called Motor Unit Action Potential (MUAP). This full electrical activity is called electromyogram (EMG) and can be recorded using electrodes located on the skin surface (SEMG). The contraction of Muscle fibres requires a train of action potentials which induces a MUAP Train (MUAPT).

Finally a MUAPT (i.e. one source) writes as a train of a specific waveform. The waveforms issued from two distinct muscles are very close as the physiological process is the same and so are not independent. Some differences may appear only if the muscle widths (and so the number of Fibers) are very different. Recall that an SEMG signal is a sparse signal composed of a train of this waveform. If the two trains are not synchronized or if a great part of the waveforms from two sources are not temporally overlapped, then a BSS method based on independence may work as proved in [2], [4], [6]. The MUAP train produces a frequency discharge which varies with the force produced by the muscle. The period of the train decreases when the force increases. It means that in the case of low force level, the sources will be less temporally overlapped than in case of high force level.

Consequently, it may appear that SEMG sources are not independent and even not uncorrelated. We cannot use the whitening step and we must find a new criterion to separate the sources. We focused on the signal nature to choose this criterion. Biomechanics studies [8] showed that in isometric contraction, a linear relation exists between the IEMG, which represents muscle activity, and the tension (or force) produced by the muscles. The IEMG is calculated from the estimated outputs by: 


$$
Y_{j}^{\mathrm{int}}(t, f)=\frac{1}{\tau} \int_{t-\tau}^{t}\left|Y_{j}(z, f)\right|^{2} d z
$$

where $Y_{j}^{\text {int }}$ is an IEMG of a $j$-th estimated sources, $\tau$ is an integration window varying between $25 \mathrm{~ms}$ and $200 \mathrm{~ms}$. Here we assume that the tension of each muscle varies independently and we propose a new separation criterion where the minimization of the covariance of the integrated SEMG replaces the independence of the SEMG.

$$
\operatorname{cov}\left(Y_{1}^{\mathrm{int}}(t, f), Y_{2}^{\mathrm{int}}(t, f)\right) \rightarrow \min .
$$

The separating matrices $\mathbf{G}(f)$ were researched by adding the constraint as follows. As we looked for normalized sources the main diagonal elements of the mixture ma$\operatorname{trix} \mathbf{H}(f)$ are ones and the secondary diagonal elements are complex numbers $h_{i j}$ with a modulus in $[0 ; 1]$. By putting ones to the main diagonal we assume that the vector of the sources is a vector of the contributions from the $i$-th source to the $i$-th electrode.

To reduce the estimation error we directly calculated the matrix $\mathbf{G}(f)$, which is inverse to $\mathbf{H}(f)$. Firstly we looked for a 2-by-2 matrix whose main diagonal elements are ones and the secondary diagonal elements are complex numbers equal to $-h_{i j}$. After finding the matrix satisfying the criterion (7) it was divided by its determinant to make this matrix inverse to $\mathbf{H}(f)$.

The minimization was performed using Nelder-Mead algorithm.

\subsection{Permutation Problem}

The main problem in a frequency approach is to resolve the permutation ambiguity. As we had only two sources, only two permutations of estimated sources are possible in each frequency bin $f$. We assume that the force profile of one source is similar in all frequency bins, hence the IEMG of each estimated source in the $f$ frequency bin is highly correlated with the IEMG of the same estimated source in the $f-1$ frequency bin. So, we choose the permutation which maximizes the sum of the covariances:

$$
\sum_{j=1}^{2} \operatorname{cov}\left(Y_{j}^{\mathrm{int}}(t, f), Y_{j}^{\mathrm{int}}(t, f-1)\right) \rightarrow \max
$$

\subsection{Source Reconstruction}

The sources $\mathbf{Y}(t, f)$ were calculated in each frequency bin by applying the corresponding separating matrix $\mathbf{G}(f)$ to the mixture $\mathbf{X}(t, f)$ in the same frequency bin.

Obtained sources were used to reconstruct the source signal using inverse STFT:

$$
\widehat{s}_{j}(t)=\operatorname{ISTFT}\left(Y_{j}(t, f)\right),
$$




\section{$3 \quad$ Results}

\subsection{Experimental Setting}

Bipolar SEMG recordings (Fig. 1) were performed as described in [2] on one male volunteer with no prior known condition or trauma on his right forearm. The SEMG signals from extensor indicis (EI) and extensor digiti minimi (EDM) were acquired during the experimental task of the extension of the index and the little finger by two pairs of electrodes, which were placed over the muscles. These two fingers have been chosen because they are known to be biomechanically independent. Five extensions were produced. The first four extensions were alternating, i.e. only one muscle was extended at the same time, and the fifth trial was a simultaneous extension of the index and little fingers. Finger extension forces were measured at the same time by the KISTLER 9017B force sensors. The SEMG and force recordings were performed by mean of the BIOPAC MP150 acquisition system. Both force and SEMG recordings were sampled synchronously at $2 \mathrm{kHz}$ with a $500 \mathrm{~Hz}$ anti-aliasing filter. After acquiring the SEMG signals were filtered forwards and backwards with an order eight Butterworth band-pass digital filer in $[20 ; 500] \mathrm{Hz}$. The force signals were filtered forwards and backwards with an order four Butterworth low-pass digital filer with $f_{s}=5 \mathrm{~Hz}$ because only low-frequency variations of the force have biomechanical application [9]. Integration time $\tau$ was fixed to $100 \mathrm{~ms}$ to calculate IEMG.
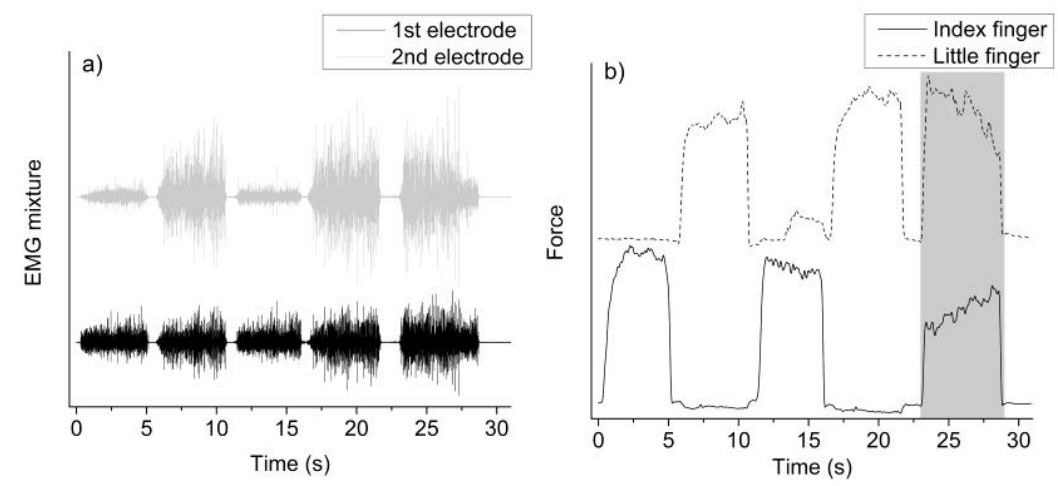

Fig. 1. The reordered SEMG signals (a) and the forces (b) of the index and the little fingers. The movement when the both fingers were active simultaneously is highlighted

\subsection{Blind Source Separation}

Two BSS algorithms, one ICA based (JADE) [7] and the algorithm based on proposed criterion (IEMG-based), were performed in frequency domain using the convolutive mixture model. BSS were performed by two different ways. At first, BSS algorithms were applied to the whole signal mixtures. Afterwards, the BSS algorithms were performed as follows. The separation matrices were calculated only for the part of the mixtures corresponding to the simultaneous extension of the index and little 
fingers, i.e. to the fifth movement (Fig. 1) and then applied to the whole signal mixtures in each frequency bin.

The permutation problem, which is typical for the frequency approach, was solved using the fact that the IEMG profiles of the reconstructed sources remained the same from one frequency bin to another (Fig. 2).

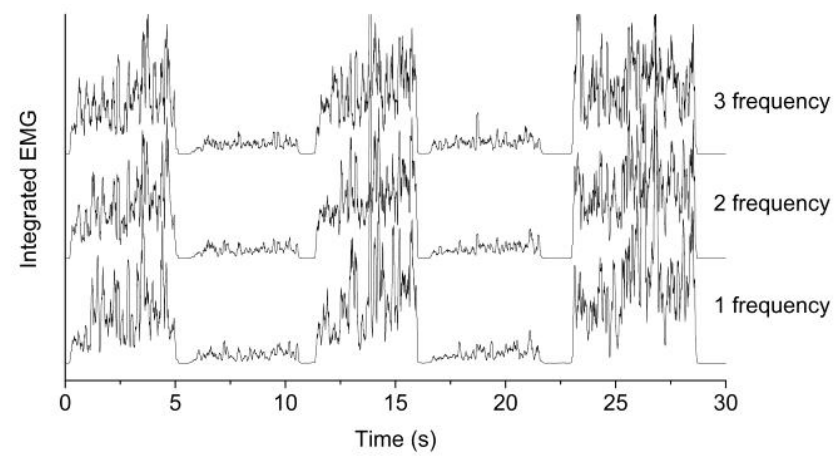

Fig. 2. The IEMG of the 3 first frequency bins of the estimated signal from extensor indicis muscle

The sources were reconstructed (Fig. 3) and used to estimate the finger forces. The crosstalk reduction efficiency was estimated as described below.

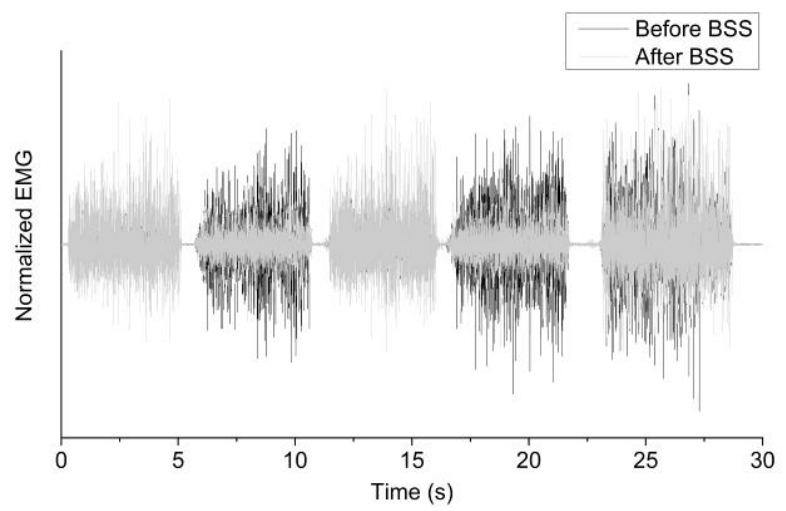

Fig. 3. The SEMG signal from extensor indicis muscle before and after BSS

\subsection{Force Estimation and Crosstalk Reduction Efficiency Measure}

The forces of EI and EDM muscles were estimated by calculating IEMG using the reconstructed source signals. The IEMG was calculated by applying (6) to the reconstructed source signals. As the force measurements, the calculated IEMG signals were filtered forwards and backwards with an order four Butterworth low-pass digital filer with $f_{s}=5 \mathrm{~Hz}$. 
Signal-to-Crosstalk Ratio. The first four trials, which are corresponding to the alternating finger movements, were used to calculate the Signal-to-crosstalk ratio (SCR). As only one finger was active at the same time during these finger movements, the signal of the source corresponding to the active muscle is a useful signal, and the signal coming from the other source could be thought as a crosstalk. The SCR was calculated as:

$$
S C R(d B)=10 \log _{10}\left(\frac{P_{1}}{P_{2}}\right),
$$

where $P_{l}$ is a power of the signal of interest and $P_{2}$ is the crosstalk.

The resulting SCR was calculated as a mean of SCRs in both initial or both reconstructed signals.

Root-Mean-Square Deviation. The RMS deviation between the measured force and the force estimated by IEMG was calculated after normalization of the measured and estimated force to unit power.

\subsection{Discussion}

The crosstalk reduction efficiencies of JADE and IEMG-based algorithm were compared for two different ways of separation matrices calculation (Table 1).

Table 1. Comparison of IEMG-based algorithm with JADE algorithm. The separation matrices were calculated from the whole mixtures and from the fifth movement

\begin{tabular}{|c|c|c|}
\hline Separation method & SCR $(\mathbf{d B})$ & RMS \\
\hline No method (signal mixture) & 5,34 & 0,74 \\
\hline \multicolumn{3}{|c|}{ The matrices $\mathbf{G}(f)$ were calculated from the whole mixtures } \\
\hline IEMG-based method & 12,98 & 0,47 \\
\hline Frequency JADE & 12,84 & 0,47 \\
\hline \multicolumn{3}{|c|}{ The matrices $\mathbf{G}(f)$ were calculated from the fifth movement } \\
\hline IEMG-based method & 11,12 & 0,54 \\
\hline Frequency JADE & 8,30 & 0,56 \\
\hline
\end{tabular}

The findings lead us to believe that the crosstalk reduction in SEMG of finger extensor muscles seems to be efficient when a convolutive mixture model is used. We can also assume that the permutation problem was properly solved for the both methods.

The performances of the both methods are close when $\mathbf{G}(f)$ were calculated from the whole mixtures, because first four movements were alternating that makes the sources unsynchronized and independent during the main part of the mixtures.

For the fifth movement the sources are strongly temporally overlapped that could make the sources no more independent, because the MUAPs' waveforms are similar. 
That may explain the decrease of JADE performance when $\mathbf{G}(f)$ were calculated from the fifth movement.

However, it would be beneficial to replicate the outlined approach for different electrode positions and for SEMG signals from other muscles.

\section{Conclusion}

We focused on the separation of FIR convolutive mixtures of Surface electromyografic signals (SEMG) to reduce the crosstalk in the SEMG signals and to improve the precision of the muscle force estimation. As independence of the SEMG sources is not always verified, we proposed to replace it by a criterion calculated on the integrated SEMG. The criterion characterizes the fact that the variations of the forces produced by the two muscles are biomechanically independent.

Acknowledgement. The authors thank Dana Lahat for her valuable comments.

\section{References}

1. Holobar, A., Farina, D.: Blind source identification from the multichannel surface electromyogram. Physiol. Meas. 35, R143-R165 (2014)

2. Léouffre, M., Quaine, F., Servière, C.: Testing of instantaneity hypothesis for blind source separation of extensor indicis and extensor digiti minimi surface electromyograms. J. Electromyogr. Kinesiol. 23, 908-15 (2013)

3. Mesin, L., Smith, S., Hugo, S., Viljoen, S., Hanekom, T.: Effect of spatial filtering on crosstalk reduction in surface EMG recordings. Med. Eng. Phys. 31, 374-383 (2009)

4. Farina, D., Févotte, C., Doncarli, C., Merletti, R.: Blind Separation of Linear Instantaneous Mixtures of Nonstationary Surface Myoelectric Signals. 51. IEEE Trans. Biomed. Eng. 51, 1555-1567 (2004)

5. Merletti, R., Holobar, A., Farina, D.: Analysis of motor units with high-density surface electromyography. J. Electromyogr. Kinesiol. 18, 879-90 (2008)

6. Jiang N., Farina D.: Covariance and Time-scale methods for blind separation of delayed sources. IEEE T. on Biomedical Engineering, vol 58, n³, 550-556 (2011)

7. Cardoso J.F, Souloumiac A.: Blind beamforming for non-gaussian signals. In: Radar and Signal Processing. IEE Proceedings F , 140, 362-370 IET (1993)

8. Lippold, O.C.J.: The relation between integrated action potentials in a human muscle and its isometric tension. J. Physiol. 117, 492-499 (1952)

9. Hoozemans, M.J.M., Van Dieën, J.H.: Prediction of handgrip forces using surface EMG of forearm muscles. J. Electromyogr. Kinesiol. 15, 358-366 (2005) 